\title{
Multiple positive solutions for Kirchhoff-Schrödinger-Poisson system with general singularity
}

\section{Qi Zhang*}

\section{"Correspondence:} zhangqi@sxu.edu.cn School of Mathematical Sciences, Shanxi University, Taiyuan, Shanxi 030006, P.R. China

\begin{abstract}
In this paper, we consider the existence of multiple positive solutions for Kirchhoff-Schrödinger-Poisson system with the nonlinear term containing both general singularity and quasicritical nonlinearity. By combining the variational method with the perturbation method, we obtain the existence of two positive solutions with the parameter $\lambda$ small enough. One of the solutions is the local minimum of the corresponding functional, and the other is the limit of the mountain pass type solution to the perturbation problem.
\end{abstract}

Keywords: Kirchhoff-Schrödinger-Poisson system; general singularity; multiple solutions; variational method; perturbation

\section{Introduction and main results}

In this paper, we are interested in discussing the existence and multiple positive solutions to the following general singular Kirchhoff-Schrödinger-Poisson system:

$$
\begin{cases}-\left(a+b \int_{\Omega}|\nabla u|^{2}\right) \Delta u+\phi u=\lambda h f(u)+g(u), & \text { in } \Omega, \\ -\Delta \phi=u^{2}, & \text { in } \Omega, \\ u>0, & \text { in } \Omega, \\ u=\phi=0, & \text { on } \partial \Omega,\end{cases}
$$

where $\Omega \subset \mathbb{R}^{3}$ is a smooth bounded domain with boundary $\partial \Omega, a>0, b \geq 0, \lambda>0$ is a parameter, $f, g, h$ satisfy the following assumptions:

(f) $f \in C\left((0, \infty), \mathbb{R}_{+}\right)$is nonincreasing and $\int_{0}^{1} f(s) d s<\infty$. Moreover, there exists $\gamma \in(0,1)$ such that

$$
\lim _{s \rightarrow 0^{+}} f(s) s^{\gamma}=\infty
$$

( $\left.g_{1}\right) g \in C\left(\mathbb{R}_{+}, \mathbb{R}_{+}\right), g(s)=o(s)$ as $s \rightarrow 0$ and $g$ has a 'quasicritical growth', namely

$$
\lim _{s \rightarrow+\infty} \frac{g(s)}{s^{5}}=0
$$

(c) The Author(s) 2017. This article is distributed under the terms of the Creative Commons Attribution 4.0 International License (http://creativecommons.org/licenses/by/4.0/), which permits unrestricted use, distribution, and reproduction in any medium, provided you give appropriate credit to the original author(s) and the source, provide a link to the Creative Commons license, and indicate if changes were made. 
(g) $\lim _{s \rightarrow+\infty} \frac{g(s)}{s^{3}}=\infty$;

(g) $g(s) s \geq 4 G(s)$, where $G(s)=\int_{0}^{s} g(t) d t, s \in \mathbb{R}_{+}$;

(h) $h \in L^{2}(\Omega)$ with $h(x)>0$ a.e. $x \in \Omega$.

Recently, the following singular Kirchhoff type problem has been studied extensively in $[1-5]$

$$
\begin{cases}-\left(a+b \int_{\Omega}|\nabla u|^{2}\right) \Delta u=\lambda h u^{-\gamma}+\mu g(x, u), & \text { in } \Omega, \\ u>0, & \text { in } \Omega, \\ u=0, & \text { on } \partial \Omega,\end{cases}
$$

where $\Omega \subset \mathbb{R}^{n}, n \geq 3$ is a smooth bounded domain with boundary $\partial \Omega, \gamma \in(0,1), \lambda, \mu \geq 0$ are parameters. In [5], by using the method of Nehari manifold, Liu and Sun discussed the existence of two positive solutions to (1.2) with $g(x, s)=g(x) \frac{s^{p}}{|x| t}, t \in[0,1), p \in[3,5-2 t)$ for the parameter $\lambda>0$ small enough. Lei, Liao and Tang in [1] combined the variational method with the perturbation argument to discuss (1.2) with $n=3$ and $g$ being critical term: $g(s)=s^{5}$ and obtained two positive solutions to this problem. In [3, 4], the authors discussed the existence and multiple positive solutions to (1.2) with $n=4$ and $g$ being critical term: $g(s)=s^{3}$. By using the Nehari manifold method and analyzing the relations between the parameters $\lambda, \mu$ and the first eigenvalue to the Kirchhoff type problem, the authors in [3] obtained multiple positive solutions to this problem. In [4], the authors obtained the existence of two positive solutions to (1.2) with $h(x)=\frac{1}{|x|^{\beta}}, \beta \in(0,3)$ by using the variational method and the perturbation method. The existence of unique positive solution to (1.2) with $g(x, s)=-s^{p}, p \in\left(0,2^{*}-1\right)$ was obtained in [2] by using the variational method. Meanwhile, the singular $(p, q)$ Kirchhoff type system was also considered in [6].

In [7], the author of the present paper considered the following singular SchrödingerPoisson system:

$$
\begin{cases}-\Delta u+\eta \phi u=\mu u^{-r}, & \text { in } \Omega, \\ -\Delta \phi=u^{2}, & \text { in } \Omega, \\ u>0, & \text { in } \Omega, \\ u=\phi=0, & \text { on } \partial \Omega,\end{cases}
$$

where $\Omega \subset \mathbb{R}^{3}$ is a smooth bounded domain with boundary $\partial \Omega, \eta= \pm 1, r \in(0,1)$ is a constant, $\mu>0$ is a parameter. The existence of unique positive solution was obtained for $\eta=1$ and any $\mu>0$ by using the variational method. The multiple positive solutions were also obtained for $\eta=-1$ and $\mu>0$ small enough by combining the variational method with the Nehari manifold method. Recently, the Schrödinger-Poisson system with singular potential was also considered in [8]. The existence of system (1.1) with $h=0, g(s)=-s^{p}$ was considered in [9].

The Kirchhoff-Schrödinger-Poisson system with general singularity $f$ and $-g(g \geq 0)$ in (1.1) was firstly considered in our recent paper [10], where $f, g, h$ satisfy the more weaker assumptions: 
$\left(\mathrm{f}_{0}\right) f \in C\left((0, \infty), \mathbb{R}_{+}\right)$satisfies that there exists $\delta>0$ such that $f$ is nonincreasing on $(0, \delta]$, $\int_{0}^{\delta} f(s) d s<\infty$, and there exist $\alpha, \gamma \in(0,1)$ such that

$$
\lim _{s \rightarrow 0^{+}} f(s) s^{\alpha}=\infty, \quad \lim _{s \rightarrow \infty} f(s) / s^{\gamma}=0 ;
$$

$\left(\mathrm{g}_{0}\right) g \in C\left(\mathbb{R}_{+}, \mathbb{R}_{+}\right)$and there exists $c_{1}>0$ such that

$$
g(s) \leq c_{1}\left(s+s^{5}\right), \quad s \in \mathbb{R}_{+}
$$

$\left(\mathrm{h}_{0}\right) h \in L^{6 /(5-\gamma)}(\Omega)$ with $h(x)>0$ a.e. $x \in \Omega$.

Under the weaker assumptions $\left(\mathrm{f}_{0}\right),\left(\mathrm{g}_{0}\right)$ and $\left(\mathrm{h}_{0}\right)$, the corresponding functional is well defined and is coercive. By using the variational method, the negative global minimum is obtained and is the unique positive solution to this problem. Based on our work [7, 10], recently, $\mathrm{Mu}$ and $\mathrm{Lu}$ in [11] considered the existence and multiplicity of positive solutions for system (1.1) with $f(s)=s^{-\gamma}, \gamma \in(0,1)$. A natural question is whether there exist multiple positive solutions to system (1.1) with the nonlinear term containing both general singular nonlinearity and the quasicritical nonlinearity.

Motivated by the above reference, especially by [1, 4, 5], and based on our work [10], in this paper, we would like to continue to study the existence of multiple solutions to the general singular Kirchhoff-Schrödinger-Poisson system (1.1).

Throughout this paper, let $H_{0}^{1}(\Omega)$ be the usual Sobolev space with the inner product and the norm

$$
(u, v)=\int_{\Omega} \nabla u \cdot \nabla v, \quad\|u\|=(u, u)^{1 / 2}, \quad u, v \in H_{0}^{1}(\Omega) .
$$

We denote the norm of $L^{p}(\Omega)$ by $|u|_{p}=\left(\int_{\Omega}|u|^{p}\right)^{1 / p}$. By the Sobolev embedding theorem, $H_{0}^{1}(\Omega)$ can be compactly embedded into $L^{p}(\Omega)$ for all $p \in[1,6)$ and the embedding $H_{0}^{1}(\Omega) \hookrightarrow L^{6}(\Omega)$ is continuous.

For any given $u \in H_{0}^{1}(\Omega)$, by using the Lax-Milgram theorem, the Dirichlet boundary problem $-\Delta \phi=u^{2}$ in $\Omega$ has a unique solution $\phi_{u} \in H_{0}^{1}(\Omega)$. Substitute $\phi_{u}$ to the first equation of system (1.1), then system (1.1) can be transformed into the following variable equation:

$$
\begin{cases}-\left(a+b \int_{\Omega}|\nabla u|^{2}\right) \Delta u+\phi_{u} u=\lambda h f(u)+g(u), & \text { in } \Omega, \\ u>0, & \text { in } \Omega, \\ u=0, & \text { on } \partial \Omega .\end{cases}
$$

Some necessary properties of $\phi_{u}$ are given in Lemma 2.1.

Since we only consider the positive solution to system (1.1), we can assume that $f(s)=0$ and $g(s)=0$ for all $s \in(-\infty, 0)$. By (f), for $s \geq 1 / 2$,

$$
F(s)=F(1 / 2)+\int_{1 / 2}^{s} f(t) d t \leq F(1 / 2)+f(1 / 2)(s-1 / 2) .
$$

Since $F(s) \leq F(1 / 2), s \in[0,1 / 2]$, then there exist $c_{1}, c_{2}>0$ such that

$$
0 \leq F(s) \leq c_{1} s+c_{2}, \quad s \in \mathbb{R}
$$


It is obvious that $F$ is continuous on $\mathbb{R}$. By $\left(\mathrm{g}_{1}\right)$, we easily obtain that for any $\varepsilon>0$, there exist $C_{\varepsilon}>0, p \in(3,5)$ such that

$$
g(s) \leq \varepsilon\left(s+s^{5}\right)+C_{\varepsilon} s^{p}, \quad s \in \mathbb{R},
$$

and

$$
G(s) \leq \varepsilon\left(s^{2}+s^{6}\right)+C_{\varepsilon} s^{p+1}, \quad s \in \mathbb{R} .
$$

Thus, by (1.4), (1.6) and (h), the energy functional corresponding to (1.3)

$$
J(u)=\frac{a}{2}\|u\|^{2}+\frac{b}{4}\|u\|^{4}+\frac{1}{4} \int_{\Omega} \phi_{u} u^{2}-\lambda \int_{\Omega} h F(u)-\int_{\Omega} G(u), \quad u \in H_{0}^{1}(\Omega)
$$

is well defined and continuous on $H_{0}^{1}(\Omega)$.

As we know, under the general singular assumption (f) or $\left(\mathrm{f}_{0}\right)$, the functional $J$ fails to be Fréchet differentiable because of the singular term. We then cannot apply the critical point theory to obtain the existence of solution directly. In general, a function $u \in H_{0}^{1}(\Omega)$ is called a solution of (1.3), that is, $\left(u, \phi_{u}\right)$ is a solution of $(1.1)$ and $u(x)>0$ a.e. in $\Omega$ satisfying

$$
\left(a+b\|u\|^{2}\right)(u, v)+\int_{\Omega} \phi_{u} u v-\lambda \int_{\Omega} h f(u) v-\int_{\Omega} g(u) v=0, \quad v \in H_{0}^{1}(\Omega) .
$$

In fact, under the weaker singular assumption $\left(f_{0}\right)$, from $(1.4),(1.6),(1.7)$, we easily deduce that the functional $J$ has a negative local minimum around the neighborhood of origin with the parameter $\lambda>0$ small enough. With the two skilled lemmas (Lemmas 2.3, 2.4 in [10]) on the properties of the singular term $f$, we can show that the negative local minimum point is a solution of problem (1.3). In order to obtain the second solution of system (1.1), here we assume that (f) holds, that is, $f$ is singular at 0 and nonincreasing on $(0, \infty)$. It is obvious that assumption (f) implies that $\left(\mathrm{f}_{0}\right)$ holds. Assumption (f) was first introduced in [12] to consider the singular semilinear elliptic equation. To obtain the second solution of problem (1.3), motivated by $[1,4]$, we also consider the perturbation problem

$$
\begin{cases}-\left(a+b\|u\|^{2}\right) \Delta u+\phi_{u} u=\lambda h f(u+\alpha)+g(u), & \text { in } \Omega, \\ u \geq 0, & \text { in } \Omega, \\ u=0, & \text { on } \partial \Omega\end{cases}
$$

where $\alpha>0$. The functional corresponding to problem (1.9) is as follows:

$$
\begin{aligned}
J_{\alpha}(u) & =\frac{a}{2}\|u\|^{2}+\frac{b}{4}\|u\|^{4}+\frac{1}{4} \int_{\Omega} \phi_{u} u^{2}-\lambda \int_{\Omega} h\left(F\left(u^{+}+\alpha\right)-F(\alpha)\right)-\int_{\Omega} G(u), \\
u & \in H_{0}^{1}(\Omega) .
\end{aligned}
$$

Under the assumptions of (f), $\left(\mathrm{g}_{1}\right)-\left(\mathrm{g}_{3}\right)$ and $(\mathrm{h})$, we can show $J_{\alpha} \in C^{1}\left(H_{0}^{1}(\Omega), \mathbb{R}\right)$ and problem (1.9) has a mountain pass type solution $u_{\alpha}$. Finally, we can prove that the limit $v_{0}$ of a family of solutions $\left\{u_{\alpha}\right\}$ of problem (1.9) is the second solution of problem (1.3). In 
the proof, the monotonic property of $f$ and a result from [13] are crucial to showing the uniform boundedness of $\left\{u_{\alpha}\right\}$ and the convergence of $u_{\alpha} \rightarrow v_{0}$ as $\alpha \rightarrow 0$.

Our main result can be described as follows.

Theorem 1.1 If $a>0, b \geq 0$, and assumptions (f), $\left(\mathrm{g}_{1}\right)-\left(\mathrm{g}_{3}\right)$ and $(\mathrm{h})$ hold, then there exists $\lambda^{*}>0$ such that system (1.1) has at least two solutions for each $\lambda \in\left(0, \lambda^{*}\right)$.

Remark 1.2 There are a number of functions which satisfy $(\mathrm{f}),\left(\mathrm{g}_{1}\right)-\left(\mathrm{g}_{3}\right),(\mathrm{h})$ respectively. For example,

(i) $f_{1}(s)=\left[s^{\alpha} \arctan (1+s)\right]^{-1}$ for all $s \in(0, \infty)$, where $0<\gamma<\alpha<1$;

(ii) $f_{2}(s)=\sqrt{1+s^{2 \beta}} / s^{\alpha}$ for all $s \in(0, \infty)$, where $0<\max \{\gamma, \beta / 2\}<\alpha<1$.

It is easy to verify that the functions $f_{1}, f_{2}$ satisfy condition (f).

Let $g_{1}(s)=s^{2+\beta} \ln (1+s), s>0$ with $\beta \in(0,1)$ and $g_{2}(s)=s^{p}, s>0$ with $p \in(3,5)$. Let $\rho \in$ $C^{1}\left(\mathbb{R}^{+},[0,1]\right)$ be a cut-off function verifying $s \rho^{\prime}(s) \leq 0,\left|\rho^{\prime}(s)\right| \leq 2$,

$$
\rho(s)= \begin{cases}1, & s \in[0,1], \\ 0, & s \in[2, \infty) .\end{cases}
$$

Set $G(s)=\rho(s) G_{1}(s)+(1-\rho(s)) G_{2}(s), g(s)=G^{\prime}(s)$, where $G_{i}(s)=\int_{0}^{s} g_{i}(t) d t$. Then it is easy to verify that $g$ satisfies conditions $\left(\mathrm{g}_{1}\right)-\left(\mathrm{g}_{3}\right)$.

Take some $x_{0} \in \Omega$ and let $h(x)=\left|x-x_{0}\right|^{-\beta}$ for all $x \in \Omega \backslash\left\{x_{0}\right\}$, where $\beta \in[0,3 / 2)$. It is obvious that $h$ satisfies condition (h).

This paper is organized as follows. In Section 2, we give the existence of a negative local minimum of the functional $J$ for $\lambda>0$ small enough and show that it is a solution of problem (1.3). In Section 3, we firstly discuss the existence of the mountain pass type solution to the perturbation problem (1.9). Furthermore, by approximation, the second solution of problem (1.3) is obtained.

In this paper, $c, c_{i}, C_{i}$ denote various positive constants, which may vary from line to line.

\section{Existence of the first solution to system (1.1)}

Let us first collect some properties of $\phi_{u}$. We refer the readers to [7, 14-16], etc.

Lemma 2.1 For each $u \in H_{0}^{1}(\Omega)$, there exists a unique solution $\phi_{u} \in H_{0}^{1}(\Omega)$ of

$$
\begin{cases}-\Delta \phi=u^{2}, & \text { in } \Omega, \\ \phi=0, & \text { on } \partial \Omega .\end{cases}
$$

The following properties hold for the solution $\phi_{u}$ :

(i) $\left\|\phi_{u}\right\|^{2}=\int_{\Omega} \phi_{u} u^{2}$;

(ii) $\phi_{u} \geq 0$. Moreover, $\phi_{u}>0$ in $\Omega$ when $u \neq 0$;

(iii) for each $t \neq 0$, it holds that $\phi_{t u}=t^{2} \phi_{u}$;

(iv) if $u_{n} \rightarrow u$ in $H_{0}^{1}(\Omega)$, then we have

$$
\phi_{u_{n}} \rightarrow \phi_{u} \quad \text { in } H_{0}^{1}(\Omega)
$$




$$
\begin{aligned}
& \int_{\Omega} \phi_{u_{n}} u_{n} \phi \rightarrow \int_{\Omega} \phi_{u} u \phi, \quad \phi \in H_{0}^{1}(\Omega), \\
& \int_{\Omega} \phi_{u_{n}} u_{n}\left(u_{n}-u\right) \rightarrow 0
\end{aligned}
$$

(v) $\phi_{u} \in W_{\mathrm{loc}}^{2,3}(\Omega) \cap C^{0}(\bar{\Omega})$;

(vi) $\phi_{u}=\phi_{u^{+}}+\phi_{u^{-}}$, where $u^{ \pm}= \pm \max \{ \pm u, 0\}$.

Under the assumptions of $(\mathrm{f}),\left(\mathrm{g}_{1}\right)$ and $(\mathrm{h})$, we can show that the functional $J$ defined in (1.7) has a negative local minimum for small $\lambda>0$. In fact, we have the following lemma.

Lemma 2.2 Under the assumptions of (f), $\left(\mathrm{g}_{1}\right)$ and $(\mathrm{h})$, there exist $\lambda^{*}>0$ and $r, \rho>0$ such that for any $\lambda \in\left(0, \lambda^{*}\right)$, we have

$$
\left.J\right|_{S_{r}} \geq \rho \quad \text { and } \quad m=\inf _{\bar{B}_{r}} J<0,
$$

where $B_{r}=\left\{u \in H_{0}^{1}(\Omega):\|u\|<r\right\}, S_{r}=\partial B_{r}$.

Proof For any $u \in H_{0}^{1}(\Omega)$, by (1.4), (1.6) with $0<\varepsilon<\frac{a}{4} \mu_{1}$, and (1.7), where $\mu_{1}>0$ is the first eigenvalue of the operator $-\Delta$ in $H_{0}^{1}(\Omega)$, we have

$$
\begin{aligned}
J(u) & \geq \frac{a}{2}\|u\|^{2}-\lambda \int_{\Omega} h\left(c_{1} u+c_{2}\right)-\int_{\Omega} \varepsilon\left(u^{2}+u^{6}\right)-C_{\varepsilon}|u|_{p+1}^{p+1} \\
& \geq \frac{a}{4}\|u\|^{2}-\lambda c_{1}|h|_{2}|u|_{2}-c_{3}\|u\|^{6}-c_{4}\|u\|^{p+1}-\lambda c_{2}|h|_{1} \\
& \geq\|u\|\left(\frac{a}{4}\|u\|-c_{3}\|u\|^{5}-c_{4}\|u\|^{p}-\lambda c_{0}|h|_{2}\right)-\lambda c_{2}|h|_{1} .
\end{aligned}
$$

Let $m(t)=\frac{a}{4} t-c_{3} t^{5}-c_{4} t^{p}$, since $p>3$, there exists $r>0$ such that $m(r)=\max _{t \geq 0} m(t)$. We choose $\lambda_{1}, \lambda_{2}>0$ respectively such that $\lambda_{1} c_{0}|h|_{2}=\frac{1}{2} m(r), \lambda_{2} c_{2}|h|_{1}=\frac{1}{4} r m(r)$. Thus, when $0<\lambda<\lambda^{*}=\min \left\{\lambda_{1}, \lambda_{2}\right\}$, for any $u \in S_{r}$, we have

$$
\begin{aligned}
J(u) & \geq r\left(m(r)-\lambda c_{0}|h|_{2}\right)-\lambda c_{2}|h|_{1} \\
& \geq \frac{1}{4} r m(r) \\
& =: \rho .
\end{aligned}
$$

Hence, for any $\lambda \in\left(0, \lambda^{*}\right)$, there exist $r, \rho>0$ such that $\left.J\right|_{S_{r}} \geq \rho$.

On the other hand, by assumption (f), there exists $\delta>0$ such that

$$
f(s) \geq s^{-\gamma}, \quad F(s) \geq \frac{s^{1-\gamma}}{1-\gamma}, \quad s \in(0, \delta] .
$$

Choose a nonnegative function $\varphi \in C_{0}^{\infty}(\Omega) \backslash\{0\}$ with $\max _{\Omega} \varphi \leq \delta$. Then, for any $t \in(0,1]$, by Lemma 2.1(iii), (2.2), we have

$$
J(t \varphi)=\frac{a t^{2}}{2}\|\varphi\|^{2}+\frac{b t^{4}}{4}\|\varphi\|^{4}+\frac{t^{4}}{4} \int_{\Omega} \phi_{\varphi} \varphi^{2}-\lambda \int_{\Omega} h F(t \varphi)-\int_{\Omega} G(t \varphi)
$$




$$
\leq \frac{a t^{2}}{2}\|\varphi\|^{2}+\frac{b t^{4}}{4}\|\varphi\|^{4}+\frac{t^{4}}{4} \int_{\Omega} \phi_{\varphi} \varphi^{2}-\frac{t^{1-\gamma}}{1-\gamma} \lambda \int_{\Omega} h \varphi^{1-\gamma}
$$

Since $1-\gamma \in(0,1)$ and $h(x)>0$, a.e. $x \in \Omega$, we get that $J(t \varphi)<0$ for $t>0$ small enough. Hence, it follows from (2.1) that $m=\inf _{\bar{B}_{r}} J<0$.

In order to prove that the local minimum $m$ can be obtained by some $u_{0} \in H_{0}^{1}(\Omega)$ and to prove that $u_{0}$ is a solution of problem (1.3), we need the following two skilled lemmas which can be found in [10].

Lemma 2.3 Assume that $\left(\mathrm{f}_{0}\right)$ holds, for $a_{0}, b_{0} \geq 0$, one has that $\lim _{t \rightarrow 0^{+}} \frac{1}{t}\left[F\left(a_{0}+t b_{0}\right)-\right.$ $\left.F\left(a_{0}\right)\right]=f\left(a_{0}\right) b_{0}$, which equals $\infty$ if $a_{0}=0$ and $b_{0}>0$.

Lemma 2.4 Assume that $\left(\mathrm{f}_{0}\right)$ holds. Then, for any $u \in H_{0}^{1}(\Omega)$ with $u(x)>0$, a.e. $x \in \Omega$, we have

$$
\lim _{t \rightarrow 0^{+}} \int_{\Omega} \frac{1}{t} h[F(u+t u)-F(u)]=\int_{\Omega} h f(u) u .
$$

Theorem 2.5 Assume that (f), $\left(\mathrm{g}_{1}\right)$ and $(\mathrm{h})$ hold. Then, for $\lambda \in\left(0, \lambda^{*}\right)$, problem (1.3) possesses a solution $u_{0}$ with $J\left(u_{0}\right)=m$.

Proof According to the definition of $m$, there exists a sequence $\left\{u_{n}\right\} \subset \bar{B}_{r}$ such that $\lim _{n \rightarrow \infty} J\left(u_{n}\right)=m$. Then $\left\{u_{n}\right\}$ is bounded in $H_{0}^{1}(\Omega)$. Going if necessary to a subsequence, still denoted by $\left\{u_{n}\right\}$, there exists $u_{0} \in H_{0}^{1}(\Omega)$ such that

$$
\begin{aligned}
& u_{n} \rightarrow u_{0} \quad \text { in } H_{0}^{1}(\Omega), \\
& u_{n} \rightarrow u_{0} \quad \text { in } L^{s}(\Omega), s \in[1,6), \\
& u_{n}(x) \rightarrow u_{0}(x), \quad \text { a.e. } x \in \Omega,
\end{aligned}
$$

as $n \rightarrow \infty$. By (1.4) and the Sobolev embedding theorem, we see that $\left\{F\left(u_{n}\right)\right\}$ is bounded in $L^{2}(\Omega)$. Moreover, it follows from the continuity of $F$ that $F\left(u_{n}(x)\right) \rightarrow F\left(u_{0}(x)\right)$, a.e. $x \in \Omega$. Thus, we obtain that $F\left(u_{n}\right) \rightarrow F\left(u_{0}\right)$ in $L^{2}(\Omega)$. By $h \in L^{2}(\Omega)$, it follows that

$$
\lim _{n \rightarrow \infty} \int_{\Omega} h F\left(u_{n}\right)=\int_{\Omega} h F\left(u_{0}\right) .
$$

By (1.6), we easily deduce $\int_{\Omega} G\left(u_{n}\right) \rightarrow \int_{\Omega} G\left(u_{0}\right)$. Then, by the weak lower semi-continuity of the norm and Lemma 2.1(iv), (2.3), we have

$$
\begin{aligned}
m & =\lim _{n \rightarrow \infty} J\left(u_{n}\right) \\
& =\liminf _{n \rightarrow \infty}\left[\frac{a}{2}\left\|u_{n}\right\|^{2}+\frac{b}{4}\left\|u_{n}\right\|^{4}+\frac{1}{4} \int_{\Omega} \phi_{u_{n}} u_{n}^{2}-\lambda \int_{\Omega} h F\left(u_{n}\right)-\int_{\Omega} G\left(u_{n}\right)\right] \\
& \geq \frac{a}{2}\left\|u_{0}\right\|^{2}+\frac{b}{4}\left\|u_{0}\right\|^{4}+\frac{1}{4} \int_{\Omega} \phi_{u_{0}} u_{0}^{2}-\lambda \int_{\Omega} h F\left(u_{0}\right)-\int_{\Omega} G\left(u_{0}\right) \\
& =J\left(u_{0}\right) .
\end{aligned}
$$


On the other hand, $u_{n} \rightarrow u_{0}$ implies that $\left\|u_{0}\right\| \leq \liminf _{n \rightarrow \infty}\left\|u_{n}\right\| \leq r$, then $J\left(u_{0}\right) \geq m$. Hence $J\left(u_{0}\right)=m$.

To show that $u_{0}$ is a solution of problem (1.3), we need to show that $u_{0}(x)>0$, a.e. $x \in \Omega$ and $u_{0}$ satisfies (1.8). The proof is similar to the proof of Theorem 1.1 in [10], for completeness, here we give the details.

By Lemma 2.1(vi), we obtain that $m \leq J\left(u_{0}^{+}\right) \leq J\left(u_{0}\right)=m$, then $J\left(u_{0}^{+}\right)=J\left(u_{0}\right)=m$. Thus we may assume $u_{0} \geq 0$. While $m<0$, then $u_{0} \neq 0$. Now we divide the proof into two steps. For convenience, we denote $l_{0}=a+b\left\|u_{0}\right\|^{2}$.

Firstly, we prove that $u_{0}(x)>0$, a.e. $x \in \Omega$. In fact, for each $v \in H_{0}^{1}(\Omega)$ with $v \geq 0$ and $t>0$ small enough, we have that

$$
\begin{aligned}
0 \leq & \frac{J\left(u_{0}+t v\right)-J\left(u_{0}\right)}{t} \\
= & \frac{a}{2 t}\left(\left\|u_{0}+t v\right\|^{2}-\left\|u_{0}\right\|^{2}\right)+\frac{b}{4 t}\left(\left\|u_{0}+t v\right\|^{4}-\left\|u_{0}\right\|^{4}\right) \\
& +\frac{1}{4 t} \int_{\Omega}\left[\phi_{u_{0}+t v}\left(u_{0}+t v\right)^{2}-\phi_{u_{0}} u_{0}^{2}\right]-\lambda \int_{\Omega} \frac{1}{t} h\left[F\left(u_{0}+t v\right)-F\left(u_{0}\right)\right] \\
& -\int_{\Omega} \frac{1}{t}\left[G\left(u_{0}+t v\right)-G\left(u_{0}\right)\right] .
\end{aligned}
$$

This implies that

$$
\liminf _{t \rightarrow 0^{+}} \lambda \int_{\Omega} \frac{1}{t} h\left[F\left(u_{0}+t v\right)-F\left(u_{0}\right)\right] \leq l_{0}\left(u_{0}, v\right)+\int_{\Omega} \phi_{u_{0}} u_{0} v-\int_{\Omega} g\left(u_{0}\right) v .
$$

Thus, by Fatou's lemma and Lemma 2.3, we have

$$
\lambda \int_{\Omega} h f\left(u_{0}\right) v \leq l_{0}\left(u_{0}, v\right)+\int_{\Omega} \phi_{u_{0}} u_{0} v-\int_{\Omega} g\left(u_{0}\right) v
$$

Now let $e_{1} \in H_{0}^{1}(\Omega)$ be the first eigenfunction of the operator $-\Delta$ in $H_{0}^{1}(\Omega)$ and $e_{1}(x)>0$ for all $x \in \Omega$. Taking $v=e_{1}$ in (2.4), one gets that

$$
\lambda \int_{\Omega} h f\left(u_{0}\right) e_{1} \leq l_{0}\left(u_{0}, e_{1}\right)+\int_{\Omega} \phi_{u_{0}} u_{0} e_{1}-\int_{\Omega} g\left(u_{0}\right) e_{1}<\infty
$$

which implies that $u_{0}(x)>0$, a.e. $x \in \Omega$ by assumption (h). If not, there exists $E \subset \Omega$ such that $m(E)>0$ and $u_{0}(x)=0$ for all $x \in E$. Then, by Lemma 2.3,

$$
\int_{\Omega} h f\left(u_{0}\right) e_{1} \geq \int_{E} h f\left(u_{0}\right) e_{1}=\infty
$$

it is a contradiction.

Secondly, we shall prove that $u_{0}$ is a solution of problem (1.3), namely, $u_{0}$ satisfies the following:

$$
l_{0}\left(u_{0}, v\right)+\int_{\Omega} \phi_{u_{0}} u_{0} v-\lambda \int_{\Omega} h f\left(u_{0}\right) v-\int_{\Omega} g\left(u_{0}\right) v=0, \quad v \in H_{0}^{1}(\Omega) .
$$


Chang Boundary Value Problems ( 2017) 2017:127

Page 9 of 17

For this purpose, we define a function $\Phi: \mathbb{R} \rightarrow \mathbb{R}$ by $\Phi(t)=J\left(u_{0}+t u_{0}\right)$, that is,

$$
\begin{aligned}
\Phi(t)= & \frac{a(1+t)^{2}}{2}\left\|u_{0}\right\|^{2}+\frac{b(1+t)^{4}}{4}\left\|u_{0}\right\|^{4}+\frac{(1+t)^{4}}{4} \int_{\Omega} \phi_{u_{0}} u_{0}^{2} \\
& -\lambda \int_{\Omega} h F\left(u_{0}+t u_{0}\right)-\int_{\Omega} G\left(u_{0}+t u_{0}\right) .
\end{aligned}
$$

Then $\Phi$ attains its local minimum at $t=0$. It follows from Lemma 2.4 that $\Phi$ is differentriable at $t=0$ and $\Phi^{\prime}(0)=0$, that is,

$$
l_{0}\left\|u_{0}\right\|^{2}+\int_{\Omega} \phi_{u_{0}} u_{0}^{2}-\lambda \int_{\Omega} h f\left(u_{0}\right) u_{0}-\int_{\Omega} g\left(u_{0}\right) u_{0}=0 .
$$

For each $v \in H_{0}^{1}(\Omega)$ and $\varepsilon>0$, let us define $v_{\varepsilon}=u_{0}+\varepsilon v$ and

$$
\Omega_{+}=\left\{x \in \Omega: u_{0}(x)+\varepsilon v(x) \geq 0\right\}, \quad \Omega_{-}=\left\{x \in \Omega: u_{0}(x)+\varepsilon v(x)<0\right\} .
$$

Then $\left.v_{\varepsilon}^{-}\right|_{\Omega_{+}}=0$ and $\left.v_{\varepsilon}^{-}\right|_{\Omega_{-}}=u_{0}+\varepsilon v$. Inserting $v_{\varepsilon}^{+}$into (2.4) and using (2.6), we obtain that

$$
\begin{aligned}
0 \leq & l_{0}\left(u_{0}, v_{\varepsilon}^{+}\right)+\int_{\Omega} \phi_{u_{0}} u_{0} v_{\varepsilon}^{+}-\lambda \int_{\Omega} h f\left(u_{0}\right) v_{\varepsilon}^{+}-\int_{\Omega} g\left(u_{0}\right) v_{\varepsilon}^{+} \\
= & l_{0}\left(u_{0}, v_{\varepsilon}\right)+\int_{\Omega} \phi_{u_{0}} u_{0} v_{\varepsilon}-\lambda \int_{\Omega} h f\left(u_{0}\right) v_{\varepsilon}-\int_{\Omega} g\left(u_{0}\right) v_{\varepsilon} \\
& -\left[l_{0}\left(u_{0}, v_{\varepsilon}^{-}\right)+\int_{\Omega} \phi_{u_{0}} u_{0} v_{\varepsilon}^{-}-\lambda \int_{\Omega} h f\left(u_{0}\right) v_{\varepsilon}^{-}-\int_{\Omega} g\left(u_{0}\right) v_{\varepsilon}^{-}\right] \\
= & \varepsilon\left[l_{0}\left(u_{0}, v\right)+\int_{\Omega} \phi_{u_{0}} u_{0} v-\lambda \int_{\Omega} h f\left(u_{0}\right) v-\int_{\Omega} g\left(u_{0}\right) v\right]-\left[l_{0} \int_{\Omega_{-}} \nabla u_{0} \cdot \nabla\left(u_{0}+\varepsilon v\right)\right. \\
& \left.+\int_{\Omega_{-}} \phi_{u_{0}} u_{0}\left(u_{0}+\varepsilon v\right)-\lambda \int_{\Omega_{-}} h f\left(u_{0}\right)\left(u_{0}+\varepsilon v\right)-\int_{\Omega_{-}} g\left(u_{0}\right)\left(u_{0}+\varepsilon v\right)\right] \\
\leq & \varepsilon\left[l_{0}\left(u_{0}, v\right)+\int_{\Omega} \phi_{u_{0}} u_{0} v-\lambda \int_{\Omega} h f\left(u_{0}\right) v-\int_{\Omega} g\left(u_{0}\right) v\right] \\
& -\varepsilon\left[l_{0} \int_{\Omega_{-}} \nabla u_{0} \cdot \nabla v+\int_{\Omega_{-}} \phi_{u_{0}} u_{0} v\right],
\end{aligned}
$$

which implies that

$$
\begin{aligned}
& l_{0} \int_{\Omega_{-}} \nabla u_{0} \cdot \nabla v+\int_{\Omega_{-}} \phi_{u_{0}} u_{0} v \\
& \quad \leq l_{0}\left(u_{0}, v\right)+\int_{\Omega} \phi_{u_{0}} u_{0} v-\lambda \int_{\Omega} h f\left(u_{0}\right) v-\int_{\Omega} g\left(u_{0}\right) v .
\end{aligned}
$$

Now let $E_{n}=\left\{x \in \Omega: u_{0}(x)>0, v(x)>-\infty, u_{0}(x)+v(x) / n<0\right\}$ for all $n$. Then $\left\{E_{n}\right\}$ is a nonincreasing sequence of measurable sets and

$$
\lim _{n \rightarrow \infty} E_{n}=\bigcap_{n=1}^{\infty} E_{n}=\emptyset .
$$


Thus we have

$$
\lim _{n \rightarrow \infty} m\left(E_{n}\right)=m\left(\lim _{n \rightarrow \infty} E_{n}\right)=0
$$

Select $\varepsilon=1 / n$. Then $\Omega_{-} \subset\left\{x \in \Omega: u_{0}(x) \leq 0\right\} \cup\{x \in \Omega: v(x)=-\infty\} \cup E_{n}$ and $m\left(\Omega_{-}\right)=$ $m\left(E_{n}\right) \rightarrow 0$ as $n \rightarrow \infty$. Letting $\varepsilon=1 / n \rightarrow 0$ in (2.7), we have

$$
0 \leq l_{0}\left(u_{0}, v\right)+\int_{\Omega} \phi_{u_{0}} u_{0} v-\lambda \int_{\Omega} h f\left(u_{0}\right) v-\int_{\Omega} g\left(u_{0}\right) v
$$

According to the arbitrariness of $v \in H_{0}^{1}(\Omega)$, this inequality also holds for $-v$. Thus, (2.5) holds. Therefore, $u_{0}$ is a solution of system (1.3) with $J\left(u_{0}\right)=m$.

\section{Proof of Theorem 1.1}

In order to overcome the difficulty caused by the singular term and to obtain the second solution of problem (1.3) for $\lambda>0$ small enough, in this section, we firstly consider the following perturbation problem:

$$
\begin{cases}-\left(a+b\|u\|^{2}\right) \Delta u+\phi_{u} u=\lambda h f(u+\alpha)+g(u), & \text { in } \Omega, \\ u \geq 0, & \text { in } \Omega, \\ u=0, & \text { on } \partial \Omega,\end{cases}
$$

where $\alpha>0$. We define the functional corresponding to problem (3.1)

$$
\begin{aligned}
J_{\alpha}(u) & =\frac{a}{2}\|u\|^{2}+\frac{b}{4}\|u\|^{4}+\frac{1}{4} \int_{\Omega} \phi_{u} u^{2}-\lambda \int_{\Omega} h\left(F\left(u^{+}+\alpha\right)-F(\alpha)\right)-\int_{\Omega} G(u), \\
u & \in H_{0}^{1}(\Omega) .
\end{aligned}
$$

It is obvious that $J_{\alpha}$ is a $C^{1}$ functional defined on $H_{0}^{1}(\Omega)$. The solution of problem (3.1) corresponds to the critical point of the functional $J_{\alpha}$. That is, if $u \in H_{0}^{1}(\Omega)$ is a solution of problem (3.1), it satisfies

$$
\begin{aligned}
& \left(a+b\|u\|^{2}\right)(u, \phi)+\int_{\Omega} \phi_{u} u \phi-\lambda \int_{\Omega} h f\left(u^{+}+\alpha\right) \phi-\int_{\Omega} g(u) \phi=0, \\
& \phi \in H_{0}^{1}(\Omega) .
\end{aligned}
$$

For any $s>0$, since $f$ is nonincreasing, we have

$$
F(s+\alpha)-F(\alpha)=\int_{\alpha}^{s+\alpha} f(t) d t=\int_{0}^{s} f(\tau+\alpha) d \tau \leq \int_{0}^{s} f(\tau) d \tau=F(s),
$$

by $F(s)=0$ if $s \leq 0,(3.3)$ holds for all $s \in \mathbb{R}$. Then, for any $u \in H_{0}^{1}(\Omega)$, we have

$$
J(u) \leq J_{\alpha}(u) \leq I(u)
$$

where $I(u)=\frac{a}{2}\|u\|^{2}+\frac{b}{4}\|u\|^{4}+\frac{1}{4} \int_{\Omega} \phi_{u} u^{2}-\int_{\Omega} G(u), u \in H_{0}^{1}(\Omega)$. 
In order to show that $J_{\alpha}$ satisfies the mountain pass geometry and to estimate the mountain pass critical level, we firstly consider the functional $I$. In fact, under the assumptions of $\left(g_{1}\right)$ and $\left(g_{2}\right)$, we have the following lemma.

Lemma 3.1 Under the assumptions of $\left(\mathrm{g}_{1}\right)$ and $\left(\mathrm{g}_{2}\right)$, there exist $r_{0}, \rho_{0}>0$ such that the functional I satisfies

(i) $\left.I\right|_{S_{r_{0}}} \geq \rho_{0}$;

(ii) there exists $u_{1} \in H_{0}^{1}(\Omega)$ with $\left\|u_{1}\right\|>r_{0}$ such that $I\left(u_{1}\right)<0$.

Proof (i) By (1.6) with $\varepsilon>0$ small enough, for any $u \in H_{0}^{1}(\Omega)$, we have

$$
I(u) \geq\left(\frac{a}{2}-c \varepsilon\right)\|u\|^{2}-c_{1}\|u\|^{6}-c_{2}\|u\|^{p+1},
$$

it is obvious that the conclusion (i) holds.

(ii) It follows from $\left(\mathrm{g}_{1}\right)$ and $\left(\mathrm{g}_{2}\right)$, for any given $M>0$, there exists $R>0$ such that $g(t) \geq$ $M t^{3}, t>R$ and

$$
\lim _{t \rightarrow 0^{+}} \frac{g(t)-M t^{3}}{t}=0 .
$$

Then there exists $C>0$ such that $g(t)-M t^{3} \geq-C t, t \in[0, R]$ and $g(t) \geq M t^{3}-C t, t \geq 0$. For $G$, we also have

$$
G(t) \geq \frac{M}{4} t^{4}-\frac{C t^{2}}{2}, \quad t \in \mathbb{R} .
$$

Thus, for any $u \in H_{0}^{1}(\Omega) \backslash\{0\}, \int_{\Omega} G(t u) \geq \frac{M}{4} t^{4}|u|_{4}^{4}-\frac{C}{2} t^{2}|u|_{2}^{2}$. It follows that

$$
\lim _{t \rightarrow \infty} \int_{\Omega} \frac{G(t u)}{t^{4}}=\infty
$$

Thus

$$
\begin{aligned}
I(t u) & =\frac{a t^{2}}{2}\|u\|^{2}+\frac{b t^{4}}{4}\|u\|^{4}+\frac{t^{4}}{4} \int_{\Omega} \phi_{u} u^{2}-\int_{\Omega} G(t u) \\
& =t^{4}\left(\frac{a}{2 t^{2}}\|u\|^{2}+\frac{b}{4}\|u\|^{4}+\frac{1}{4} \int_{\Omega} \phi_{u} u^{2}-\int_{\Omega} \frac{G(t u)}{t^{4}}\right),
\end{aligned}
$$

it follows from (3.5) that $\lim _{t \rightarrow \infty} I(t u)=-\infty$, hence, there exists $t>0$ large enough such that $\left\|u_{1}\right\|=\|t u\|>r_{0}$ and $I\left(u_{1}\right)<0$.

Now, we define

$$
\Gamma=\left\{\gamma \in C\left([0,1], H_{0}^{1}(\Omega)\right): \gamma(0)=0, \gamma(1)=u_{1}\right\}, \quad c=\inf _{\gamma \in \Gamma \in[0,1]} \max _{t} I(\gamma(t)) .
$$

It follows from Lemma 3.1 that $c \geq \rho_{0}>0$. For any given $\alpha>0, J_{\alpha}$ also has the mountain pass geometry. In fact, we have the following lemma. 
Lemma 3.2 Assume $\lambda \in\left(0, \lambda^{*}\right)$, under the assumptions of $(\mathrm{f}),\left(\mathrm{g}_{1}\right),\left(\mathrm{g}_{2}\right)$ and $(\mathrm{h})$, for $r, \rho>0$ (where $\lambda^{*}, r, \rho$ are given in Lemma 2.2), the functional $J_{\alpha}$ satisfies the following:

(i) $\left.J_{\alpha}\right|_{S_{r}} \geq \rho$;

(ii) there exists $v \in H_{0}^{1}(\Omega)$ such that $J_{\alpha}(v)<0$.

Proof (i) By (3.4) and Lemma 2.2, the conclusion holds.

(ii) From (3.4) and (ii) of Lemma 3.1, we choose $v=u_{1}$ in Lemma 3.1 and the conclusion holds.

We also can define the mountain pass critical level

$$
c_{\alpha}=\inf _{\gamma \in \Gamma} \max _{t \in[0,1]} J_{\alpha}(\gamma(t))
$$

From (3.4) and (i) of Lemma 3.2, for $\lambda \in\left(0, \lambda^{*}\right)$,

$$
0<\rho \leq c_{\alpha} \leq c
$$

In the following, we give the existence of the mountain pass type solution to system (3.1).

Lemma 3.3 Suppose that (f), $\left(\mathrm{g}_{1}\right)-\left(\mathrm{g}_{3}\right)$ and $(\mathrm{h})$ hold, $\lambda \in\left(0, \lambda^{*}\right)$. Then there exists $u_{\alpha} \in$ $H_{0}^{1}(\Omega)$ such that

$$
J_{\alpha}^{\prime}\left(u_{\alpha}\right)=0, \quad J_{\alpha}\left(u_{\alpha}\right)=c_{\alpha} .
$$

Proof By Lemma 3.2 and the mountain pass lemma, there exists a sequence $\left\{u_{n}\right\} \subset H_{0}^{1}(\Omega)$ such that $J_{\alpha}\left(u_{n}\right) \rightarrow c_{\alpha}, J_{\alpha}^{\prime}\left(u_{n}\right) \rightarrow 0$. By (3.3), (1.4) and $\left(\mathrm{g}_{3}\right)$, for $n$ large enough, we have

$$
\begin{aligned}
c_{\alpha}+1+\left\|u_{n}\right\| \geq & J_{\alpha}\left(u_{n}\right)-\frac{1}{4}\left(J_{\alpha}^{\prime}\left(u_{n}\right), u_{n}\right) \\
= & \frac{a}{4}\left\|u_{n}\right\|^{2}-\lambda \int_{\Omega} h\left(F\left(u_{n}^{+}+\alpha\right)-F(\alpha)-\frac{1}{4} f\left(u_{n}^{+}+\alpha\right) u_{n}\right) \\
& -\int_{\Omega}\left(G\left(u_{n}\right)-\frac{1}{4} g\left(u_{n}\right) u_{n}\right) \\
\geq & \frac{a}{4}\left\|u_{n}\right\|^{2}-\lambda \int_{\Omega} h F\left(u_{n}^{+}\right)+\frac{\lambda}{4} f(\alpha) \int_{\Omega} h u_{n}^{-} \\
\geq & \frac{a}{4}\left\|u_{n}\right\|^{2}-\lambda^{*}\left(c_{0}|h|_{2}\left\|u_{n}\right\|+c_{2}\left|h_{1}\right|+\frac{1}{4} f(\alpha) c_{0}|h|_{2}\left\|u_{n}\right\|\right) .
\end{aligned}
$$

Then $\left\{u_{n}\right\}$ is bounded. Up to a subsequence, there exists $u_{\alpha} \in H_{0}^{1}(\Omega)$ such that $u_{n} \rightarrow u_{\alpha}$ in $H_{0}^{1}(\Omega)$ and

$$
\begin{aligned}
& u_{n} \rightarrow u_{\alpha} \quad \text { in } L^{s}(\Omega), s \in[1,6), \\
& u_{n}(x) \rightarrow u_{\alpha}(x) \quad \text { a.e. } x \in \Omega,
\end{aligned}
$$

there exists $k_{1} \in L^{2}(\Omega)$ such that for all $n,\left|u_{n}(x)\right|,\left|u_{\alpha}(x)\right| \leq k_{1}(x)$ a.e. in $\Omega$. 
It follows from $J_{\alpha}^{\prime}\left(u_{n}\right) \rightarrow 0$ that

$$
\begin{aligned}
0 \leftarrow & \left(J_{\alpha}^{\prime}\left(u_{n}\right), u_{n}-u_{\alpha}\right) \\
= & \left(a+b\left\|u_{n}\right\|^{2}\right)\left(u_{n}, u_{n}-u_{\alpha}\right)+\int_{\Omega} \phi_{u_{n}} u_{n}\left(u_{n}-u_{\alpha}\right) \\
& -\lambda \int_{\Omega} h f\left(u_{n}^{+}+\alpha\right)\left(u_{n}-u_{\alpha}\right)-\int_{\Omega} g\left(u_{n}\right)\left(u_{n}-u_{\alpha}\right) .
\end{aligned}
$$

Since $h f\left(u_{n}^{+}+\alpha\right)\left(u_{n}-u_{\alpha}\right) \rightarrow 0$ a.e. in $\Omega$ and

$$
\left|h f\left(u_{n}^{+}+\alpha\right)\left(u_{n}-u_{\alpha}\right)\right| \leq 2 f(\alpha) h k_{1} \in L^{1}(\Omega)
$$

by the dominated convergence theorem, we have

$$
\int_{\Omega} h f\left(u_{n}^{+}+\alpha\right)\left(u_{n}-u_{\alpha}\right) \rightarrow 0, \quad \text { as } n \rightarrow \infty .
$$

By (1.5), we can deduce that

$$
\begin{aligned}
\int_{\Omega} g\left(u_{n}\right)\left(u_{n}-u_{\alpha}\right) \leq & \varepsilon\left(\left|u_{n}\right|_{2}\left|u_{n}-u_{\alpha}\right|+\left|u_{n}\right|_{6}^{5}\left|u_{n}-u\right|_{6}\right) \\
& +C_{\varepsilon}\left|u_{n}\right|_{p+1}^{p}\left|u_{n}-u\right|_{p+1} \rightarrow 0,
\end{aligned}
$$

and

$$
\int_{\Omega} g\left(u_{n}\right) u_{n} \rightarrow \int_{\Omega} g\left(u_{\alpha}\right) u_{\alpha}, \quad \int_{\Omega} g\left(u_{n}\right) \phi \rightarrow \int_{\Omega} g\left(u_{\alpha}\right) \phi, \quad \forall \phi \in H_{0}^{1}(\Omega) .
$$

From (3.7), using (3.8), (3.9), Lemma 2.1(iv) and the boundedness of $\left\{u_{n}\right\}$, we get $\left\|u_{n}\right\| \rightarrow$ $\left\|u_{\alpha}\right\|$. This combined with $u_{n} \rightarrow u_{\alpha}$ implies that $u_{n} \rightarrow u_{\alpha}$ in $H_{0}^{1}(\Omega)$. Consequently, we have $J_{\alpha}\left(u_{\alpha}\right)=c_{\alpha}>\rho, J_{\alpha}^{\prime}\left(u_{\alpha}\right)=0$, that is, $u_{\alpha}$ is a nontrivial solution to problem (3.1). Then $u_{\alpha}$ satisfies (3.2), taking the test function $\phi=u_{\alpha}^{-}$in (3.2), it follows that $\left\|u_{\alpha}^{-}\right\|=0$. Thus, we have $u_{\alpha} \geq 0, u_{\alpha} \neq 0$ and $J_{\alpha}\left(u_{\alpha}\right)=c_{\alpha} \geq \rho_{1}$. Hence, by the strong maximum principle, $u_{\alpha}$ is a positive solution of the perturbation problem (3.1).

In order to consider the convergence of $\left\{u_{\alpha}\right\}$ as $\alpha \rightarrow 0$ and to obtain the second solution of problem (1.3), we need the following result, which can be found in [13].

Lemma 3.4 (Brezis and Nirenberg [13]) Let $\Omega$ be a bounded domain in $\mathbb{R}^{n}$ with smooth boundary $\partial \Omega$. Let $u \in L_{\mathrm{loc}}^{1}(\Omega)$ and assume that, for some $k \geq 0$, $u$ satisfies, in the sense of distributions,

$$
\begin{cases}-\Delta u+k u \geq 0, & \text { in } \Omega \\ u \geq 0, & \text { in } \Omega .\end{cases}
$$

Then either $u \equiv 0$, or there exists $C>0$ such that

$$
u(x) \geq C \operatorname{dist}(x, \partial \Omega), \quad x \in \Omega
$$


Remark 3.5 By Lemma 3.3, (3.2) and Lemma 2.1(v), we have

$$
-\Delta u_{\alpha}+K u_{\alpha} \geq-\Delta u_{\alpha}+\frac{\phi_{u_{\alpha}} u_{\alpha}}{a+b\left\|u_{\alpha}\right\|^{2}}=\frac{\lambda h f\left(u_{\alpha}+\alpha\right)+g\left(u_{\alpha}\right)}{a+b\left\|u_{\alpha}\right\|^{2}} \geq 0, \quad x \in \Omega,
$$

where $K>0$. Then, by Lemma 3.4, there exists $C>0$ such that $u_{\alpha}(x) \geq C \operatorname{dist}(x, \partial \Omega), x \in \Omega$.

Finally, let $\alpha \rightarrow 0$, we shall prove that the limit of a family of solutions $\left\{u_{\alpha}\right\}$ of the perturbation problem (3.1) is the second solution of problem (1.3) with $\lambda \in\left(0, \lambda^{*}\right)$, where $\lambda^{*}$ is defined in Lemma 2.2.

Theorem 3.6 Suppose that $(\mathrm{f}),\left(\mathrm{g}_{1}\right)-\left(\mathrm{g}_{3}\right)$ and $(\mathrm{h})$ hold, $\lambda \in\left(0, \lambda^{*}\right)$. Then problem (1.3) has a solution $v_{0}$ satisfying $J\left(v_{0}\right)>0$.

Proof Let $\alpha \rightarrow 0$ and $u_{\alpha} \geq 0$ is the solution of problem (3.1), that is, $J_{\alpha}\left(u_{\alpha}\right)=c_{\alpha}, J_{\alpha}^{\prime}\left(u_{\alpha}\right)=0$. Then, by $\left(\mathrm{g}_{3}\right)$, (3.3), (3.6) and (1.4), we have

$$
\begin{aligned}
c>c_{\alpha}= & J_{\alpha}\left(u_{\alpha}\right)-\frac{1}{4}\left(J_{\alpha}^{\prime}\left(u_{\alpha}\right), u_{\alpha}\right) \\
= & \frac{a}{4}\left\|u_{\alpha}\right\|^{2}-\lambda \int_{\Omega} h\left(F\left(u_{\alpha}+\alpha\right)-F(\alpha)-\frac{1}{4} f\left(u_{\alpha}+\alpha\right) u_{\alpha}\right) \\
& -\int_{\Omega}\left(G\left(u_{\alpha}\right)-\frac{1}{4} g\left(u_{\alpha}\right) u_{\alpha}\right) \\
\geq & \frac{a}{4}\left\|u_{\alpha}\right\|^{2}-\lambda \int_{\Omega} h\left(F\left(u_{\alpha}\right)\right) \\
\geq & \frac{a}{4}\left\|u_{\alpha}\right\|^{2}-\lambda^{*}\left(c_{0}|h|_{2}\left\|u_{\alpha}\right\|+c_{2}|h|_{1}\right),
\end{aligned}
$$

then $\left\{u_{\alpha}\right\}$ is bounded in $H_{0}^{1}(\Omega)$. Up to a subsequence, there exists $v_{0} \in H_{0}^{1}(\Omega)$ such that $u_{\alpha} \rightarrow v_{0}$ in $H_{0}^{1}(\Omega)$ and

$$
\begin{aligned}
& u_{\alpha} \rightarrow v_{0} \quad \text { in } L^{s}(\Omega), s \in[1,6), \\
& u_{\alpha}(x) \rightarrow v_{0}(x) \quad \text { a.e. } x \in \Omega,
\end{aligned}
$$

there exists $k_{2} \in L^{2}(\Omega)$ such that for all $n,\left|u_{\alpha}(x)\right|,\left|v_{0}(x)\right| \leq k_{2}(x)$ a.e. in $\Omega$.

Firstly, we show that $v_{0}(x)>0$ a.e. in $\Omega$. For that purpose, we denote $w_{\alpha}=u_{\alpha}-v_{0}$ and $l=\lim _{\alpha \rightarrow 0}\left\|w_{\alpha}\right\|$. Taking $\phi \in H_{0}^{1}(\Omega)$ with $\phi \geq 0$ in (3.2), we have

$$
\lambda \int_{\Omega} h f\left(u_{\alpha}+\alpha\right) \phi=\left(a+b\left\|u_{\alpha}\right\|^{2}\right)\left(u_{\alpha}, \phi\right)+\int_{\Omega} \phi_{u_{\alpha}} u_{\alpha} \phi-\int_{\Omega} g\left(u_{\alpha}\right) \phi .
$$

By using Fatou's lemma, Lemma 2.1(iv) and (3.10), we have

$$
\lambda \int_{\Omega} h f\left(v_{0}\right) \phi \leq\left(a+b l^{2}+b\left\|v_{0}\right\|^{2}\right)\left(v_{0}, \phi\right)+\int_{\Omega} \phi_{v_{0}} v_{0} \phi-\int_{\Omega} g\left(v_{0}\right) \phi .
$$

Similar to the proof of $u_{0}(x)>0$ in $x \in \Omega$ in Theorem 2.5, we can show that $v_{0}(x)>0$ a.e. in $\Omega$. 
Next, we show that $u_{\alpha} \rightarrow v_{0}$ in $H_{0}^{1}(\Omega)$ and $v_{0}$ is the solution of problem (1.3), that is, we need to show that $l=0$ and $v_{0}$ satisfies (1.8).

We take $\phi \in C_{0}^{\infty}(\Omega)$ with supp $\phi=\Omega_{1} \Subset \Omega$ in (3.2). By Remark 3.5, for $x \in \Omega_{1}$, we have

$$
\begin{aligned}
\left|h f\left(u_{\alpha}+\alpha\right) \phi\right| & \leq\left|h f\left(u_{\alpha}\right) \phi\right| \\
& \leq|h f(\operatorname{dist}(x, \partial \Omega)) \phi| \\
& \leq\left|h f\left(k_{0}\right) \phi\right| \in L^{1}(\Omega),
\end{aligned}
$$

where $k_{0}=\min _{x \in \Omega_{1}} \operatorname{dist}(x, \partial \Omega)>0$. Since $h f\left(u_{\alpha}+\alpha\right) \phi \rightarrow h f\left(v_{0}\right) \phi$ a.e. in $\Omega$, then by the dominant convergence theorem, we have

$$
\int_{\Omega} h f\left(u_{\alpha}+\alpha\right) \phi \rightarrow \int_{\Omega} h f\left(v_{0}\right) \phi \quad \text { as } \alpha \rightarrow 0 .
$$

By $\left(J_{\alpha}^{\prime}\left(u_{\alpha}\right), \phi\right)=0$, using $\int_{\Omega} \phi_{u_{\alpha}} u_{\alpha} \phi \rightarrow \int_{\Omega} \phi_{v_{0}} v_{0} \phi$ and (3.10), we get that

$$
\begin{aligned}
& \left(a+b l^{2}+b\left\|v_{0}\right\|^{2}\right)\left(v_{0}, \phi\right)+\int_{\Omega} \phi_{v_{0}} v_{0} \phi=\lambda \int_{\Omega} h f\left(v_{0}\right) \phi+\int_{\Omega} g\left(v_{0}\right) \phi, \\
& \forall \phi \in C_{0}^{\infty}(\Omega) .
\end{aligned}
$$

Since $C_{0}^{\infty}(\Omega)$ is dense in $H_{0}^{1}(\Omega)$, then for $\phi \in H_{0}^{1}(\Omega)$, there exists a sequence $\left\{\phi_{n}\right\} \subset$ $C_{0}^{\infty}(\Omega)$ such that $\phi_{n} \rightarrow \phi$ as $n \rightarrow \infty$. For $n, m \in \mathbb{N}$ large enough, replacing $\phi$ with $\phi_{n}-\phi_{m}$ in (3.11), we obtain that

$$
\begin{gathered}
\left(a+b l^{2}+b\left\|v_{0}\right\|^{2}\right)\left(v_{0}, \phi_{n}-\phi_{m}\right)+\int_{\Omega} \phi_{\nu_{0}} v_{0}\left(\phi_{n}-\phi_{m}\right) \\
=\lambda \int_{\Omega} h f\left(v_{0}\right)\left(\phi_{n}-\phi_{m}\right)+\int_{\Omega} g\left(v_{0}\right)\left(\phi_{n}-\phi_{m}\right) .
\end{gathered}
$$

Since $\phi_{n} \rightarrow \phi$, from (3.12), we can deduce that $\left\{h f\left(v_{0}\right) \phi_{n}\right\}$ is a Cauchy sequence in $L^{1}(\Omega)$, hence there exists $v \in L^{1}(\Omega)$ satisfying $h f\left(v_{0}\right) \phi_{n} \rightarrow v$ in $L^{1}(\Omega)$, which means that $h f\left(v_{0}\right) \phi_{n} \rightarrow v$ in measure. By Riesz's theorem, $\left\{h f\left(v_{0}\right) \phi_{n}\right\}$ has a subsequence, still denoted by $\left\{h f\left(v_{0}\right) \phi_{n}\right\}$, such that $h f\left(v_{0}\right) \phi_{n} \rightarrow v$ a.e. in $\Omega$. On the other hand, $h f\left(v_{0}\right) \phi_{n} \rightarrow h f\left(v_{0}\right) \phi$ a.e. in $\Omega$. So $v=h f\left(v_{0}\right) \phi$, that is, $\int_{\Omega} h f\left(v_{0}\right) \phi_{n} \rightarrow \int_{\Omega} h f\left(v_{0}\right) \phi$ as $n \rightarrow \infty$. Then, taking the test function $\phi_{n}$ in (3.11) and passing to the limit as $n \rightarrow \infty$, we obtain that (3.11) holds for any $\phi \in H_{0}^{1}(\Omega)$. We take $\phi=v_{0}$ in (3.11) and obtain that

$$
\left(a+b l^{2}+b\left\|v_{0}\right\|^{2}\right)\left\|v_{0}\right\|^{2}+\int_{\Omega} \phi_{\nu_{0}} v_{0}^{2}=\lambda \int_{\Omega} h f\left(v_{0}\right) v_{0}+\int_{\Omega} g\left(v_{0}\right) v_{0} .
$$

On the other hand, by $\left(J_{\alpha}^{\prime}\left(u_{\alpha}\right), u_{\alpha}\right)=0$, we have

$$
\left(a+b\left\|u_{\alpha}\right\|^{2}\right)\left\|u_{\alpha}\right\|^{2}+\int_{\Omega} \phi_{u_{\alpha}} u_{\alpha}^{2}=\lambda \int_{\Omega} h f\left(u_{\alpha}+\alpha\right) u_{\alpha}+\int_{\Omega} g\left(u_{\alpha}\right) u_{\alpha} .
$$

Since $f$ is nonincreasing on $(0, \infty)$, we have

$$
s f(s) \leq \int_{0}^{s} f(t) d t=F(s), \quad s \geq 0 .
$$


Then, by (1.4), we have

$$
h f\left(u_{\alpha}+\alpha\right) u_{\alpha} \leq h f\left(u_{\alpha}\right) u_{\alpha} \leq h F\left(u_{\alpha}\right) \leq h\left(c_{1}\left|u_{\alpha}\right|+c_{2}\right) \leq h\left(c_{1} k_{2}+c_{2}\right) \in L^{1}(\Omega) .
$$

Then, combining with $h f\left(u_{\alpha}+\alpha\right) \rightarrow h f\left(v_{0}\right) v_{0}$ a.e. in $\Omega$ and using the dominant convergence theorem, we have

$$
\int_{\Omega} h f\left(u_{\alpha}+\alpha\right) u_{\alpha} \rightarrow \int_{\Omega} h f\left(v_{0}\right) v_{0}
$$

Thus, from (3.14), by (3.10) and Lemma 2.1(iv), we obtain that

$$
\left(a+b l^{2}+b\left\|v_{0}\right\|^{2}\right)\left(l^{2}+\left\|v_{0}\right\|^{2}\right)+\int_{\Omega} \phi_{v_{0}} v_{0}^{2}=\lambda \int_{\Omega} h f\left(v_{0}\right) v_{0}+\int_{\Omega} g\left(v_{0}\right) v_{0} .
$$

Combining with (3.13) and (3.15), we get

$$
\left(a+b l^{2}+b\left\|v_{0}\right\|^{2}\right) l^{2}=0
$$

from $a>0, b \geq 0$, it implies that $l=0$, that is, $u_{\alpha} \rightarrow v_{0}$. Hence, from (3.11) with $l=0$ and $\phi \in H_{0}^{1}(\Omega)$, we get that $v_{0}$ is the solution of problem $(1.3)$ for $\lambda \in\left(0, \lambda^{*}\right)$ and $J\left(v_{0}\right)=$ $\lim _{\alpha \rightarrow 0} J_{\alpha}\left(u_{\alpha}\right) \geq \rho>0$.

Proof of Theorem 1.1 By Theorems 2.5 and 3.6, for $\lambda \in\left(0, \lambda^{*}\right)$, there exist two solutions $u_{0}, v_{0} \in H_{0}^{1}(\Omega)$ to problem $(1.3)$ with $J\left(v_{0}\right)>0>J\left(u_{0}\right)$, that is, system (1.1) possesses at least two solutions for each $\lambda \in\left(0, \lambda^{*}\right)$.

\section{Conclusion}

In this paper, by using the variational method and the perturbation method, we consider the existence and multiple solutions to the singular Kirchhoff-Schrödinger-Poisson system (1.1). The nonlinear terms contain the quasicritical nonlinearity $g$, which satisfies assumptions $\left(\mathrm{g}_{1}\right)-\left(\mathrm{g}_{3}\right)$, and the general singularity $f$, which satisfies $(\mathrm{f})$. The general singular assumption derives from our previous work [10], in which we consider the uniqueness of solution to Kirchhoff-Schrödinger-Poisson system. Therefore, the results in this paper are the continuation of our research in [10]. Our results also improve the results in [11], in which the authors considered the existence of Kirchhoff-Schrödinger-Poisson system with the singular term $f(s)=s^{-r}, r \in(0,1)$.

\section{Acknowledgements}

The author thanks the anonymous referee for the careful reading and some helpful comments

Funding

The paper is supported by the National Natural Science Foundation of China (Grant No. 11571209, 11671239), Science Council of Shanxi Province (2015021007), Scientific and Technological Higher Education Institutions in Shanxi (2016106).

Competing interests

The author declares that they have no competing interests. 


\section{Publisher's Note}

Springer Nature remains neutral with regard to jurisdictional claims in published maps and institutional affiliations.

Received: 15 March 2017 Accepted: 15 August 2017 Published online: 29 August 2017

\section{References}

1. Lei, C-Y, Liao, J-F, Tang, C-L: Multiple positive solutions for Kirchhoff type of problems with singularity and critical exponents. J. Math. Anal. Appl. 421(1), 521-538 (2015)

2. Liao, J-F, Ke, X-F, Lei, C-Y, Tang, C-L: A uniqueness result for Kirchhoff type problems with singularity. Appl. Math. Lett. 59, 24-30 (2016)

3. Liao, J-F, Zhang, P, Liu, J, Tang, C-L: Existence and multiplicity of positive solutions for a class of Kirchhoff type problems with singularity. J. Math. Anal. Appl. 430(2), 1124-1148 (2015)

4. Liu, R-Q, Tang, C-L, Liao, J-F, Wu, X-P: Positive solutions of Kirchhoff type problem with singular and critical nonlinearities in dimension four. Commun. Pure Appl. Anal. 15(5), 1841-1856 (2016)

5. Liu, X, Sun, Y: Multiple positive solutions for Kirchhoff type problems with singularity. Commun. Pure Appl. Anal. 12(2), 721-733 (2013)

6. Rasouli, SH: Existence of solutions for singular $(p, q)$-Kirchhoff type systems with multiple parameters. Electron. J. Differ. Equ. 2016, 69 (2016)

7. Zhang, Q: Existence, uniqueness and multiplicity of positive solutions for Schrödinger-Poisson system with singularity. J. Math. Anal. Appl. 437(1), 160-180 (2016)

8. Jiang, Y, Zhou, H-S: Schrödinger-Poisson system with singular potential. J. Math. Anal. Appl. 417(1), 411-438 (2014)

9. Mugnai, D: Schrödinger-Poisson system with positive potential. Commun. Partial Differ. Equ. 36, 1099-1117 (2011)

10. Li, F, Song, Z, Zhang, Q: Existence and uniqueness results for Kirchhoff-Schrödinger-Poisson system with general singularity. Appl. Anal. (2016). doi:10.1080/00036811.2016.1253065

11. Mu, M, Lu, H: Existence and multiplicity of positive solutions for Schrödinger-Kirchhoff-Poisson system with singularity. J. Funct. Spaces 2017, Article ID 5985962 (2017)

12. Lair, AV, Shaker, AW: Classical and weak solutions of a singular semilinear elliptic problem. J. Math. Anal. Appl. 211(2), 371-385 (1997)

13. Brezis, H, Nirenberg, L: $H^{1}$ versus $C^{1}$ local minimizers. C. R. Math. Acad. Sci. 317(5), 465-472 (1993)

14. Benci, V, Fortunato, D: An eigenvalue problem for the Schrödinger-Maxwell equations. Topol. Methods Nonlinear Anal. 11(2), 283-293 (1998)

15. D’Aprile, T, Mugnai, D: Solitary waves for nonlinear Klein-Gordon-Maxwell and Schrödinger-Maxwell equations. Proc. R. Soc. Edinb. A 134(5), 893-906 (2004)

16. Ruiz, D: The Schrödinger-Poisson equation under the effect of a nonlinear local term. J. Funct. Anal. 237(2), 655-674 (2006)

\section{Submit your manuscript to a SpringerOpen ${ }^{\circ}$ journal and benefit from:}

- Convenient online submission

- Rigorous peer review

Open access: articles freely available online

- High visibility within the field

- Retaining the copyright to your article

Submit your next manuscript at $\boldsymbol{~ s p r i n g e r o p e n . c o m ~}$ 